\title{
Research on Quanzhou Arts and Crafts Resource Library Based on 3D Printing Platform
}

\author{
Wang Xinghe ${ }^{1}$ \\ ${ }^{1}$ School of Cultural Communication, Liming Vocational University, Quanzhou, Fujian 362000, China
}

\begin{abstract}
Objective:Under the background of attaching importance to the arts and crafts industry, this paper will explore the ideas and methods for the development of the Quanzhou City Arts and Crafts Resource library based on the 3D printing platform. Method: From the perspective of analyzing the general situation of the arts and crafts in Quanzhou and the advantages of the 3D printing-based development process, the three aspects of the construction content, the innovation and the cross-industry derivative nature of the resource library were described. Conclusion:For the development of traditional arts and crafts industrialization, digitization is the only way to go. Quanzhou's arts and crafts resource library based on 3D printing platform not only realizes digitalization, but also provides a reference for the development of other regional arts and crafts resource libraries .
\end{abstract}

\section{Introduction}

In 2017, since the Ministry of Culture, the Ministry of Industry and Information Technology and the Ministry of Finance jointly issued the "Chinese Traditional Crafts Revitalization Plan", provincial and municipal governments at all levels have actively promoted various policies, and the heritage of traditional crafts and fine arts has been well protected, and the output value has also increased significantly. Taking Quanzhou as an example, as the five traditional industries of arts and crafts, in 2018 , the sales output value was more than 102.1 billion yuan, with an annual growth rate of $13.2 \%$, breaking the 100 billion yuan mark for the first time [1]. However, the stable development of the arts and crafts industry also has many serious problems to be solved, such as slow product development, high cost, unmanned succession of traditional craftsmanship, too slow manual production methods, etc.

At present, there is no resource library on the market that integrates arts and crafts into the 3D printing platform. The research group relies on cutting-edge 3D printing technology to assist the transformation and upgrading of traditional industries, and proposes the concept of three-dimensional online big data platforms for crafts. By integrating three technologies of threedimensional scanning, digital sculpture, and 3D printing samples into the traditional arts and crafts industry, it can greatly optimize the intelligent manufacturing production process in the arts and crafts industry to meet the needs of designers, 3D printing companies, and handicraft factories, which has noteworthy market prospects.

\section{Quanzhou City Arts and Crafts Overview and Advantages}

Arts and crafts is one of the five traditional industries and the ten cultural industries of Quanzhou. The Quanzhou Municipal Party Committee and Municipal Government have always attached great importance to the development of the arts and crafts industry [2], especially since the "Twelfth Five-Year Plan" period, many effective measures have been taken to fully explore advantages and deepen the implementation of the project drive and brand strategy encouraged enterprises to carry out technological and artistic innovation, and the arts and crafts industry showed a steady and relatively rapid growth trend. In 2018, there were more than 6,000 craft products enterprises in Quanzhou, with more than 400,000 employees. Among them, 383 craft enterprises above designated size have completed sales of more than 102.1 billion yuan [1], breaking the 100 billion yuan mark for the first time, which has become another one hundred billion industrial cluster in Quanzhou after textile and clothing, footwear, petrochemical, building materials and household, machinery and equipment, food and beverage, etc. Large-scale arts and crafts industrial clusters such as stone jade carving, ceramics, rattan and iron have gradually formed with a certain scale of the industry. On July 10, 2018, Quanzhou was awarded the title of "Quanzhou, the Capital of Chinese Arts and Crafts", and the regional characteristics are very obvious. Table 1 shows the honorary titles of arts and crafts awarded to Quanzhou. 
Table 1 Honorary titles of arts and crafts awarded in Quanzhou

\begin{tabular}{cl}
\hline No. & Runs \\
\hline 1 & Huian \\
2 & Dehua \\
3 & Anxi \\
4 & Fengze \\
5 & Licheng \\
6 & Yongchun \\
\\
Advantages of the 3D printing- \\
development process
\end{tabular}

The development of arts and crafts can be divided into the traditional development process and the 3D printingbased development process, both of which have their advantages and disadvantages, as the comparison of the two craft development processesshown in Table 2. By the comparison, it can be concluded that the 3D printingbased crafts development process realizes the process of re-innovation from data scanning, data modification, and innovative product printing through the combination of reverse engineering, rapid prototyping technology and

Appellation

Stone Carving Capital Of The World

Ceramic Capital Of The World

Chinese Rattan Iron Crafts Capital

Chinese Resin Crafts Capital

Chinese Folk Crafts Capital

Chinese Fragrant City

intelligent manufacturing of arts and crafts. Its independent model making can savedevelopment costs and cycles, which have huge advantages for satisfying mass production of products. At the same time, 3D printing uses rapid prototyping and additive manufacturing technology, which is a new type of digital manufacturing technology. In 2017, the scale of China's $3 \mathrm{D}$ printing manufacturing industry has reached 8 billion yuan, and it has grown at a compound annual growth rate of $26.4 \%$. [3] While the price of 3D printers is getting cheaper, the types of $3 \mathrm{D}$ printers available are also increasing many. Therefore, for the development of traditional arts and crafts industrialization, digitalization, mechanization, and intelligence are the only way to go.

Table 2 Relative comparison of crafts development process

\begin{tabular}{cccc}
\hline No. & Type & Process & Pros and cons \\
\hline 1 & $\begin{array}{c}\text { Traditional } \\
\text { development process }\end{array}$ & $\begin{array}{c}\text { Clay sculpture } \rightarrow \text { rolling over } \rightarrow \\
\text { prototyping } \rightarrow \text { hand carving }\end{array}$ & $\begin{array}{c}\text { With manual value, but slow } \\
\text { speed and inaccurate }\end{array}$ \\
2 & $\begin{array}{c}\text { 3D printing-based } \\
\text { development process }\end{array}$ & $\begin{array}{c}\text { 3D scanning / modeling } \rightarrow \text { data } \\
\text { modification } \rightarrow \text { 3D printing / data } \\
\text { programming engraving machine } \\
\text { productdirectly }\end{array}$ & $\begin{array}{c}\text { Fast speed, mass production, } \\
\text { high accuracy }\end{array}$ \\
\hline
\end{tabular}

\section{Resource library construction plan for integrating arts and crafts into 3D printing platform}

\subsection{Contents of the construction of the resource library}

3D printing in the arts and crafts industry can generally be divided into three processes, as shown in Figure 1, but the actual coreactually is digitalization. The construction of a three-dimensional display resource library of "Internet + Big Data" crafts with regional industry characteristics and influence mainly includes three aspects: online 3D printing service, digital material library, and personalized customization.

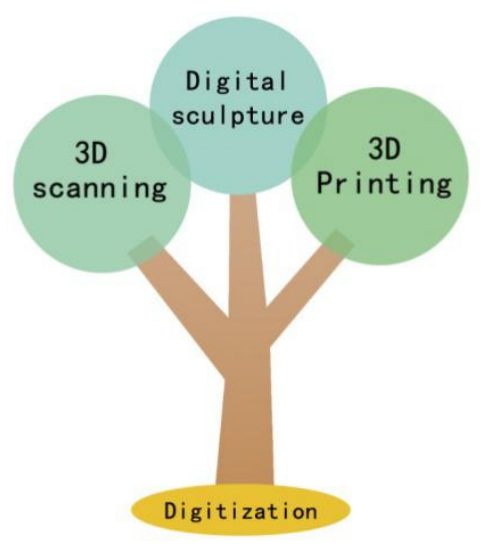

Figure 1 Development process based on 3D printing 
(1) Online 3D printing service

The 3D printing service process in the resource library includes the four steps of uploading the model, selecting materials and suppliers, smart quoting, and online payment, which mainly includes three parts including 3D file uploading, selecting materials, and selecting suppliers. The 3D file upload is to convert 3D print data into STL format for upload, as shown in Figure 2 ; the choice of materials mainly includes resin (ordinary)
$48 \mathrm{H}$, resin (ordinary) $12 \mathrm{H}$, resin (transparent), red wax and other materials. Different materials will have corresponding pictures, as shown in Figure 3; the choice of suppliers can be based on praise, star rating, number of orders, provinces and cities, as shown in Figure 4; When the data is uploaded and the materials are confirmed with the supplier, the price will be displayed and customers can choose according to their needs.

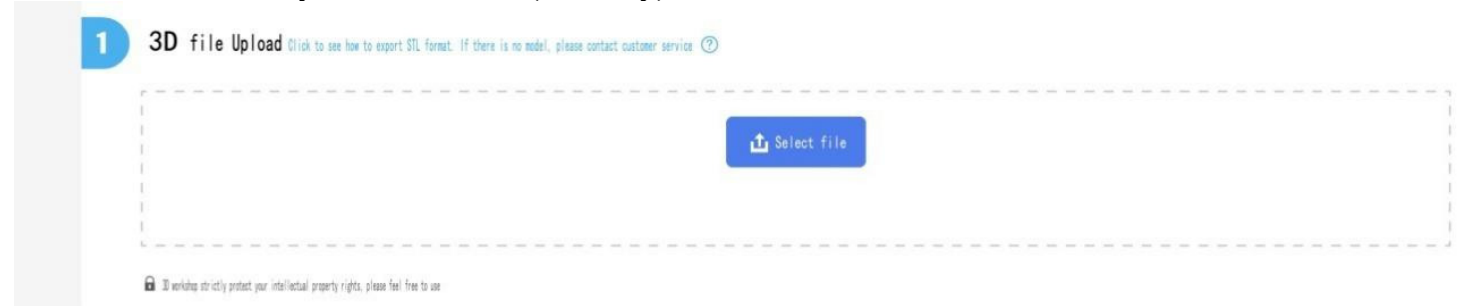

Figure 2 3D file upload

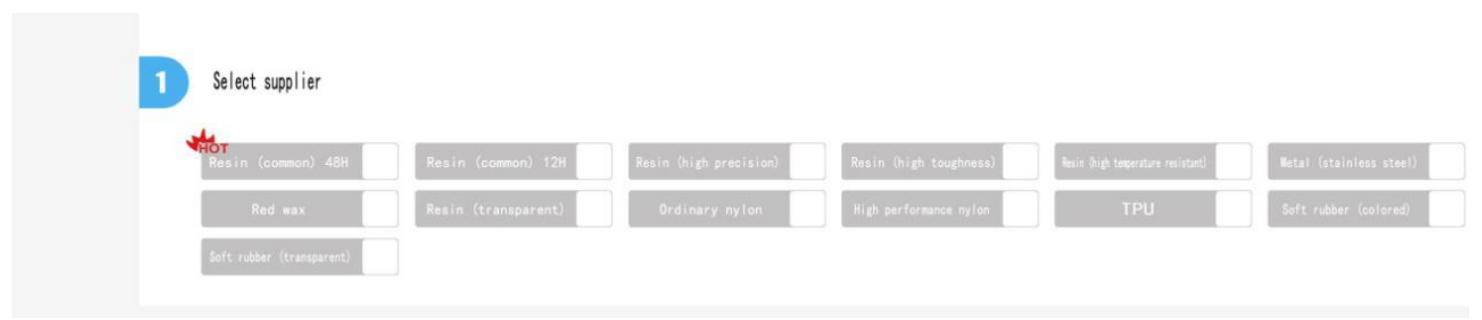

Figure 3 Selection of different materials

3

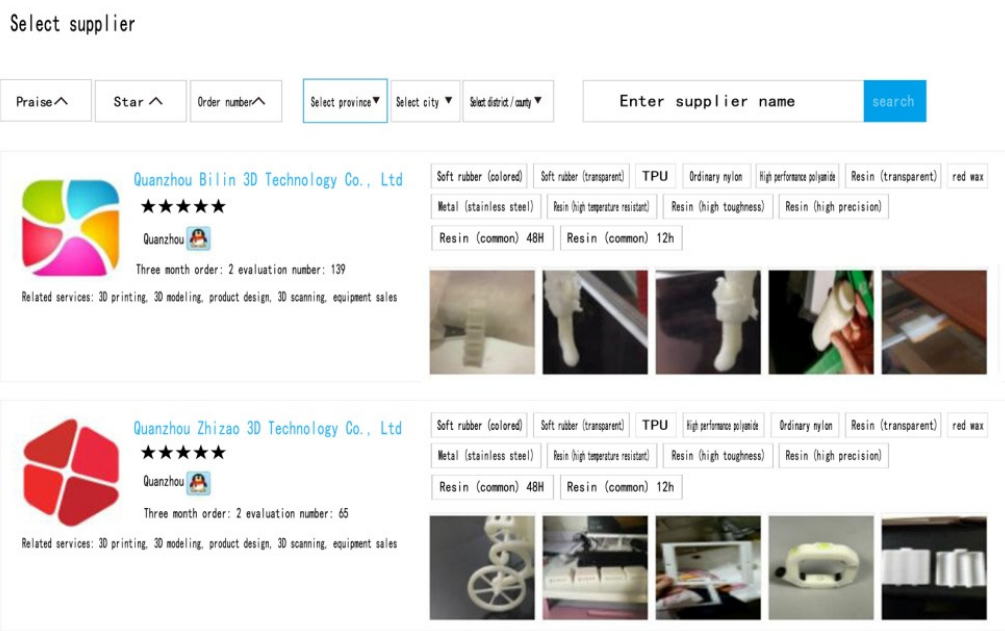

Figure 4 Selection of different suppliers

(2) Digital Material Library

The collection of digital three-dimensional data is mainly classified by the relatively large-scale arts and crafts industry clusters in Quanzhou City, including stone carving, ceramics, rattan, resin, folk crafts, incense and other six categories. There are two main ways: One is industry collecting or purchasing three-dimensional data of industrial products in batches, and then summarizing and organizing them. Another is that, related arts and crafts companies provide paid data services on the platform while scanning the three-dimensional data of existing products to save storage space in the model warehouse.

(3) Personalized customization

In terms of consumers, based on the $3 \mathrm{D}$ printing platform of Quanzhou Arts and Crafts Resource Library, consumers can choose their favorite 3D handicraft models to print or propose related design directions, which is conducive to the conversion of cultural resources into cultural production factors, avoiding the disconnect between technology and art.

In terms of designers, the Quanzhou Arts and Crafts Resource Library based on the 3D printing platform will provide designers with more economic benefits and 
attract more designers to invest in the platform.

In terms of $3 \mathrm{D}$ printing companies, due to the increase in product data in CPS smart terminals, it is beneficial for enterprises to promote 3D printers, which has a certain role in demonstration and economic benefits.

\subsection{Innovations in the Resource Library}

Based on the 3D printing platform, the Quanzhou Arts and Crafts Resource Library combines reverse engineering and rapid prototyping technology with the intelligent manufacturing of arts and crafts, realize the process of re-innovation from data scanning and data modification, printing of innovative products, independent model production, saves development costs and cycles, and ensures the smooth progress of product production. Its innovation is shown in Figure 5.

(1) Massive development related model libraries can be downloaded directly [4], making it easier for arts and crafts enterprises to develop (differentfrom other resource platforms, the $3 \mathrm{D}$ database can be downloaded and used, which is directly related to development and has higher user stickiness).

(2) Based on the massive model library, convenient secondary development and cross-development of different categories can reduce development costs, improve development efficiency, and increase the speed of new product updates.

(3) It is a full digital process, model-programmingmachine carving, realizes mechanization and intelligence, which can greatly increase the speed of mass production. (Skip the traditional clay modeling process, and the digital model developed by the product can be directly transferred to the mass production of the engraving machine.

(4) It can be associated crowdsourcing 3D printing samples, so that the models developed by the enterprise can be directly $3 \mathrm{D}$ printed, making the products more precise.

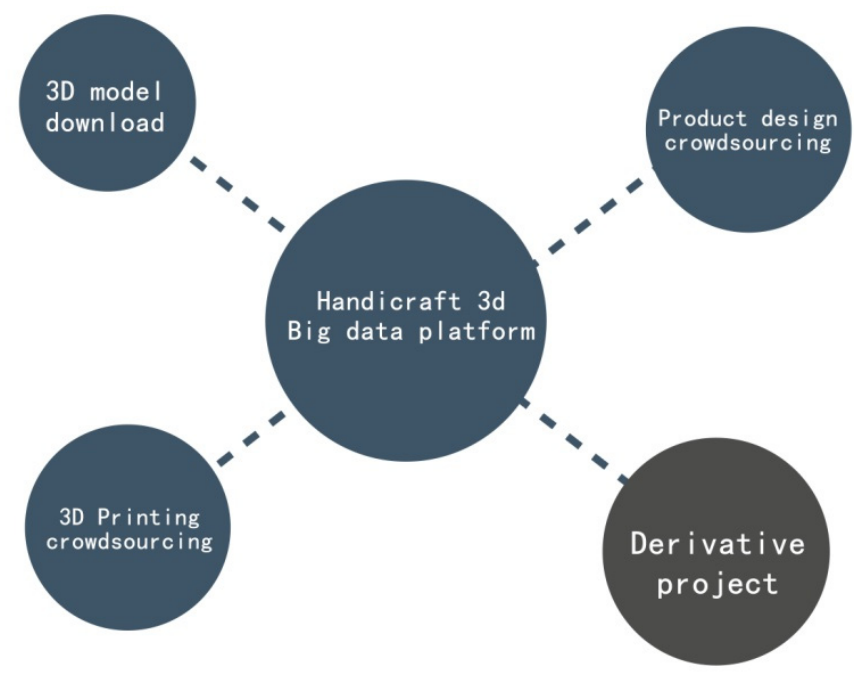

Figure 5 Resource Library Features

\subsection{Derivativeness of resource pools across industries}

In the future, 3D printing technology will participate in the production of static models, physical effects simulation, mechanical models, and post-movie product development in film and television production. The 3D printing platform-based Quanzhou Arts and Crafts Resource Library has good cross-industry derivatives and can create more value and better user stickiness.

(1) Animation games: 3D models of different craft industries can be directly supplied to the industry for basic models or secondary development, which can reduce the amount of time spent in forward modeling and greatly improve the efficiency of product updates [5].

(2) VR: The VR industry will develop at a high speed in $5 \mathrm{G}$ bandwidth acceleration, and the fastest way to obtain models in VR scenes is to access 3D scanning and existing 3D model libraries.

(3) Film and television production: More and more film and television works use a large number of $\mathrm{CG}$ computer special effects lenses, and a large number of various types of art and craft model libraries can be directly used as materials. The $3 \mathrm{D}$ printing prototype does not require the characteristics of mold opening and mass production. In actual shooting, the production road has inherent advantages. First of all, the production process is short, and there is almost no limit to complexity; The combination of 3D scanning and 3D printing facilitates data collection.Last, the 3D printed miniature model has a high degree of reduction, especially for close-up lenses. Through physical special effects and digital special effects, alternate use of telescopic and wide-angle lenses, so that the whole film will be more textured.

\section{Conclusion}

Quanzhou's arts and crafts are developing rapidly and the regional characteristics are obvious. Based on the $3 \mathrm{D}$ 
printing platform, the research and development of the Quanzhou arts and crafts resource library is based on digital technology, and 3D printing is based on the online 3D printing service, digital material library, and personalized customization. While promoting the industrial development of the arts and crafts industry in Quanzhou, related practices have certain reference significance for the construction of similar resource library projects.

\section{Acknowledgments}

Fund Project: The Social Science Planning Project of Quanzhou City (2019H33); The Youth and Youth Discipline (Professional) Leader Training Program of Quanzhou Higher School.

\section{References}

1. Quanzhou Statistical Information Network [EB/OL].(2019) http://tjj.quanzhou.gov.cn/tjzl/tjsj/ndsj/201905/P020 190521425595992585.html.

2. Zheng ,Y.F.(2018) Development of "Master Economy" Quanzhou vigorously promotes the construction of a strong cultural city [N/OL]. Quanzhou Evening News, 2013-07-6. http://qz.fjsen.com/201307/06/content_11834737.htm.

3. W, Q. (2018) Development Status and Trend Analysis of Chinese Additive Manufacturing Industry . J. China Technology Industry, 2: 54-45.

4. Wang,X.H. (2015) Tourism Creative Product Design Based on Quanzhou Regional Culture . J . Journal of Liming Vocational University, 2:87

5. Zhao,D.C. (2017) Regional imageryextraction of ancient artifacts and regionalinnovative design of products. J . Packaging Engineering, 38. 